\title{
Choroidal morphology and short-term outcomes of combination photodynamic therapy in polypoidal choroidal vasculopathy
}

\author{
Jiwon Baek $\mathbb{D}^{1} \cdot$ Jae Hyung Lee ${ }^{2} \cdot$ Sohee Jeon $^{2} \cdot$ Won Ki Lee ${ }^{2}$
}

Received: 12 January 2018 / Revised: 20 June 2018 / Accepted: 2 July 2018 / Published online: 10 October 2018

(c) The Royal College of Ophthalmologists 2018

\begin{abstract}
Purpose To investigate outcomes of combined photodynamic therapy (PDT) and intravitreal bevacizumab in association with choroidal morphology in polypoidal choroidal vasculopathy (PCV).

Method Eighty-six PCV eyes (83 patients) treated with PDT in combination with intravitreal bevacizumab and followed for 1 year were evaluated. Choroidal morphological features including subfoveal choroidal thicknesses, diameter of pachyvessel, and choroidal vascularity were analyzed for association with responsiveness and recurrence.

Result Total choroid, Haller's layer, and pachyvessel were thicker in responders $(n=70)$ compared with non-responders $(n$ $=16$ ) at baseline ( 298 vs. $227 \mu \mathrm{m}, 213$ vs. $144 \mu \mathrm{m}$, and 276 vs. $210 \mu \mathrm{m}$, respectively; all $P \leq 0.001$ ). Choroidal vascularity was significantly higher $(0.68$ vs $0.60, P<0.001)$ and choroidal hyperpermeability was more frequent in responders $(44$ vs $13 \%, P=0.018)$. Significant thinning of total choroid was observed in both responders and non-responders at 3 months after combination PDT (both $P<0.05$ ), but the reduction was greater in responders $(-33 \mu \mathrm{m}$ vs. $-10 \mu \mathrm{m}, P=0.036$ ). In recurrent eyes $(n=26)$, increase in pachyvessel diameter and choroidal vascularity was observed at recurrence.

Conclusion Choroidal morphology including characteristic features of pachychoroid and high vascularity can serve as predictive factors for outcomes after combination PDT in eyes with PCV.
\end{abstract}

\section{Introduction}

As the introduction of polypoidal choroidal vasculopathy (PCV) by Yannuzzi et al. [1] and Spaide et al. [2], our knowledge of the disease has increased considerably. Spectral-domain optical coherence tomography (OCT) has revealed that PCV is a variant of type 1 choroidal neovascularization $(\mathrm{CNV})$, which is located between the retinal pigment epithelium band and the outer part of Bruch's membrane [3]. In recent years, the choroidal features of the disease were evaluated using enhanced depth imaging (EDI), swept-source, and en-face OCT [4-7]. It is now widely accepted that PCV belongs to pachychoroid

Won Ki Lee, M.D., Ph.D.

wklee@ catholic.ac.kr

1 Department of Ophthalmology and Visual Science, Bucheon St. Mary's Hospital, College of Medicine, The Catholic University of Korea, Gyeonggi-do, Korea

2 Department of Ophthalmology and Visual Science, Seoul St. Mary's Hospital, College of Medicine, The Catholic University of Korea, Seoul, Korea spectrum diseases, which show not only the absolute thickening of the choroid but also distinctive choroidal features with dilated outer vessels and attenuated choriocapillaris [4, 8-10]. However, controversy still remains whether the entire PCV could fall into the same category.

There were attempts to classify PCV into subtypes based on clinical characteristics [11,12]. Difference in subfoveal choroidal thickness between groups was one of the common findings of those studies. Response to treatment also differed according to clinical phenotypes. Eyes with choroidal vascular hyperpermeability are known to show lower response rate to anti-vascular endothelial growth factor (anti-VEGF) therapy and thicker choroid [6, 13-15]. Based on above findings, the role of the choroid could be important in the pathogenesis of PCV and may also exert some influence on the response to photodynamic therapy (PDT) in combination with anti-VEGF.

Although intravitreal anti-VEGF injection is the mainstream treatment for neovascular age-related macular degeneration (AMD), a large portion of neovascular AMD that responds poorly to anti-VEGF was reported to be PCV. In addition, anti-VEGF treatment shows limited efficacy in terms of polyp regression in PCV [16-20]. PDT combined 
with anti-VEGF therapy seems to achieve optimal results in anatomical and functional improvement [20,21]. A metaanalysis of anti-VEGF, PDT, and combination therapy revealed that combination therapy shows a synergistic effect in terms of polyp regression and maintaining or improving visual acuity [22]. Therefore, PDT still remains as a viable treatment option for PCV.

Predicting the response to treatment would enable optimal decisions on the treatment modality in PCV patients in whom both anti-VEGF and PDT can be considered as the first-line therapy. As the response to treatment is associated with the underlying pathogenesis of a disease and the choroid plays an important role in PCV, we analyzed the clinical factors governing the outcomes of combination PDT in terms of initial response and recurrence, focusing on choroidal features.

\section{Materials and methods}

\section{Patients}

This is a retrospective, comparative, and interventional case study. We conducted a review of the medical records of patients who underwent verteporfin (Visudyne; Novartis AG, Basel, Switzerland) PDT in combination with bevacizumab for PCV between November 2011 and September 2014 at Seoul St. Mary's Hospital of the Catholic University of Korea. The study was approved by the Institutional Review Board of the Catholic Medical Center.

A diagnosis of PCV was established by the presence of polypoidal dilatation, with or without a branching vascular network (BVN) on ICGA using a confocal scanning laser ophthalmoscope (Heidelberg Retina Angiograph 2; Heidelberg Engineering, Heidelberg, Germany). Inclusion criteria were eyes affected by PCV with macular fluid and/or hemorrhage involving the fovea and received PDT in combination with intravitreal bevacizumab, which was followed for at least 1 year and no previous anti-VEGF treatment within the past 3 months. Exclusion criteria were any coexisting ophthalmic pathology, any treatment history with PDT or laser, any ocular surgery performed within the past 6 months or the use of systemic medications that might have affected choroidal thickness (e.g., steroids) and a second PDT within 1 year. Eyes that developed massive subretinal or vitreous hemorrhage after PDT were also excluded.

All patients received combination therapy consisting of a single injection of $1.25 \mathrm{mg}$ intravitreal bevacizumab (Avastin; Genentech Inc, San Francisco, CA, USA) and a session of PDT 3 days after the injection. Regarding PDT application, all patients received a $6 \mathrm{mg} / \mathrm{m}^{2}$ body surface infusion of verteporfin over $10 \mathrm{~min}$. PDT at standard fluence (light dose, $50 \mathrm{~J} / \mathrm{cm}^{2}$; dose rate, $600 \mathrm{~mW} / \mathrm{cm}^{2}$; wavelength, $689 \mathrm{~nm}$ ) was applied for $83 \mathrm{~s} 15 \mathrm{~min}$ after the start of the infusion. The laser spot size was derived by adding 1000 $\mu \mathrm{m}$ to the greatest linear dimension of the lesion. Patients were followed monthly for 3 months after PDT and regularly at 1 - to 3 -month intervals thereafter, depending on lesion activity. No additional treatment was performed during the first 3-month follow-up period unless there was a sign of recurrence.

Comprehensive ocular examinations, including bestcorrected visual acuity (BCVA) testing, fundus examination with slit-lamp biomicroscopy, auto-refractometry (KR1, Topcon Corporation, Tokyo, Japan), spectral-domain OCT (SD-OCT) imaging (Spectralis; Heidelberg Engineering) with an EDI protocol, fluorescence angiography, and ICGA at baseline were assessed in all patients. Snellen visual acuity was converted to the logarithm of the minimal angle of resolution (logMAR) for statistical analysis. Central macular thickness of the central $1 \mathrm{~mm}$ zone was measured automatically using a Spectralis OCT system and height of pigment epithelium detachment (PED) was measured manually on OCT images using Heidelberg Eye Explorer software (v.6.0.9.0; Heidelberg Engineering). Number of polyps and greatest linear dimension of lesion was measured manually on ICGA image using Heidelberg Eye Explorer software. Choroidal vascular hyperpermeability, defined as multifocal areas of hyperfluorescence with blurred margins within the choroid in the mid to late phase of ICGA, was assessed [23]. BCVA and SD-OCT were obtained at each follow-up visits.

\section{Assessment of response and recurrence after PDT}

Treatment response was determined anatomically using OCT. Responders were defined as those who showed complete resolution of intraretinal and subretinal fluid in the absence of expanding PED on OCT 3 months after PDT. Non-responders were those who showed increased persistent intraretinal and/or subretinal fluid on OCT 3 months after PDT. Recurrence was defined as reappearance of intraretinal and/or subretinal fluid on OCT after a complete resorption of fluids in responders. If there was persistent, increased or recurrent intraretinal and/or subretinal fluid after PDT, additional intravitreal injections of $0.5 \mathrm{mg}$ ranibizumab (Lucentis; Genentech) were administered using pro re nata regimens until OCT showed a dry macula.

\section{Choroidal thickness and vascularity measurements}

Two independent graders (J.B. and J.H.L.) experienced in analyzing OCT images performed choroidal thickness measurements of the total choroid and each layer. SFCT, defined as the vertical distance between the hyperreflective 
line of Bruch membrane and the choroidoscleral border, was measured using the horizontal and vertical line scans intersecting the center of the fovea. And then, the thickness of Haller's layer was measured largely based upon a previously described method $[10,24]$. In addition, by analyzing EDI-OCT raster line scans and serial ICGA images, pachyvessels (i.e., prominently dilated vessels in Haller's layer under the disease foci) below the area in or around which the BVN or polypoidal structure (in cases without $\mathrm{BVN}$ ) filling originated were identified. The luminal diameter of pachyvessel at the thickest point of the pachyvessel area was measured.

Choroidal vascularity at the area of PDT was also assessed using previously describe method by Sonoda et al. [25] Binarization of the transverse cross sectional subfoveal OCT image was done by a modified Niblack method using Image $\mathrm{J}$ (version 1.47; provided in the public domain by the National Institutes of Health, Bethesda, MD, USA; http:// imagej.nih.gov/ij/). The region of interest included was only the area of the subfoveal choroid that covers the range of PDT application (Fig. 1).

The choroidal thickness measurements were obtained at baseline and 3 months after combination PDT. The average measurements of the two observers were used for analysis. Pachyvessel thickness and choroidal vascularity measurements were obtained at baseline and 3, 6, and 12 months in non-recurrent cases and at recurrence in recurrent cases.

\section{Statistical analyses}

Statistical analyses were performed using SPSS statistical software version 19.0 (SPSS Inc., Chicago, IL, USA). Comparisons between groups were made using the $\chi^{2}$-test for categorical variables and Student's $t$ test for continuous variables after normal distribution confirmation using the Kolmogorov-Smirnov test. The Mann-Whitney $U$ test was used when normal distribution was not confirmed. The paired $t$ test was used for comparison of choroidal thickness between baseline and 3 months after PDT in each group. Repeated-measures analysis of variance was used to confirm the differences in pachyvessel diameter and choroidal vascularity change after PDT between groups. $P<0.05$ was considered statistically significant.

\section{Results}

\section{Patient baseline characteristics}

Of 92 eyes with PCV, which were followed for $>1$ year after combination PDT, 3 eyes, which developed massive hemorrhage after PDT and 3 eyes, which received the second PDT within 1 year were excluded. In total, 86 eyes of 83 patients affected by PCV were enrolled in the study. The mean patient age was $66.5 \pm 6.2$ years (range, 52-83 years) and 59 patients (61 eyes, $71 \%$ ) were male. The mean logMAR BCVA was $0.41 \pm 0.33$ (range, 0-1.7, Snellen equivalent of 20/51). The mean central macular thickness and the greatest linear dimention of the lesion was $370 \pm$ $103 \mu \mathrm{m}$ (range, $141-843 \mu \mathrm{m}$ ) and $2473 \pm 742 \mu \mathrm{m}$ (range, 1034-4735 $\mu \mathrm{m})$, respectively. PED was present in 77 eyes $(90 \%)$ and the mean height was $267.9 \pm 151.6 \mu \mathrm{m}$ (range, $50-805 \mu \mathrm{m})$. Lesions were located at macula in 84 eyes $(98 \%)$ and peripapillary in 2 eyes $(2 \%)$.

\section{The overall treatment outcome}

Complete resolution of fluid was seen in 70 eyes $(81 \%$, responders) and 16 eyes (19\%, non-responders) showed persistent fluid 3 months after combination PDT. Among 70 responders, recurrence during the 1-year follow-up was seen in 26 eyes (37\%, recurrent group) and 44 eyes (63\%, non-recurrent group) remained dry for 1 year after combination PDT.

The mean CMT at 3 months after PDT was thicker in non-responders, but CMT at 6 and 12 months in nonresponders did not differ between groups with adjunctive anti-VEGF $(369 \pm 109 \mu \mathrm{m}, 262 \pm 98 \mu \mathrm{m}, 273 \pm 76 \mu \mathrm{m}$, and $281 \pm 74 \mu \mathrm{m}$ in responders vs. $378 \pm 70 \mu \mathrm{m}, 355 \pm 89 \mu \mathrm{m}$, $324 \pm 85 \mu \mathrm{m}$, and $348 \pm 107 \mu \mathrm{m}$ in non-responders at baseline, 3, 6, and 12 months, respectively. $P=0.211,0.004$, 0.574, and 0.084). LogMAR BCVA also was better in responders at 3 months, but it did not differ between groups at 6 and 12 months $(0.40 \pm 0.33,0.34 \pm 0.29,0.33 \pm 0.28$, and $0.31 \pm 0.29$ in responders vs. $0.50 \pm 0.30,0.51 \pm 0.30$, $0.43 \pm 0.27$, and $0.39 \pm 0.25$ in non-responders at baseline, 3,6 , and 12 months, respectively. $P=0.277,0.043,0.184$, and 0.369).

\section{Comparison of baseline characteristics between groups}

Baseline clinical and morphological characteristics of responders vs. non-responders and recurrent vs. nonrecurrent eyes are compared in Table 1. Choroidal vascular hyperpermeability was more frequent in responders. There was no significant difference in baseline factors between recurrent and non-recurrent group.

\section{Comparison of choroidal thicknesses between groups}

Baseline choroidal morphologies and their changes at 3 months after PDT are compared between groups in 


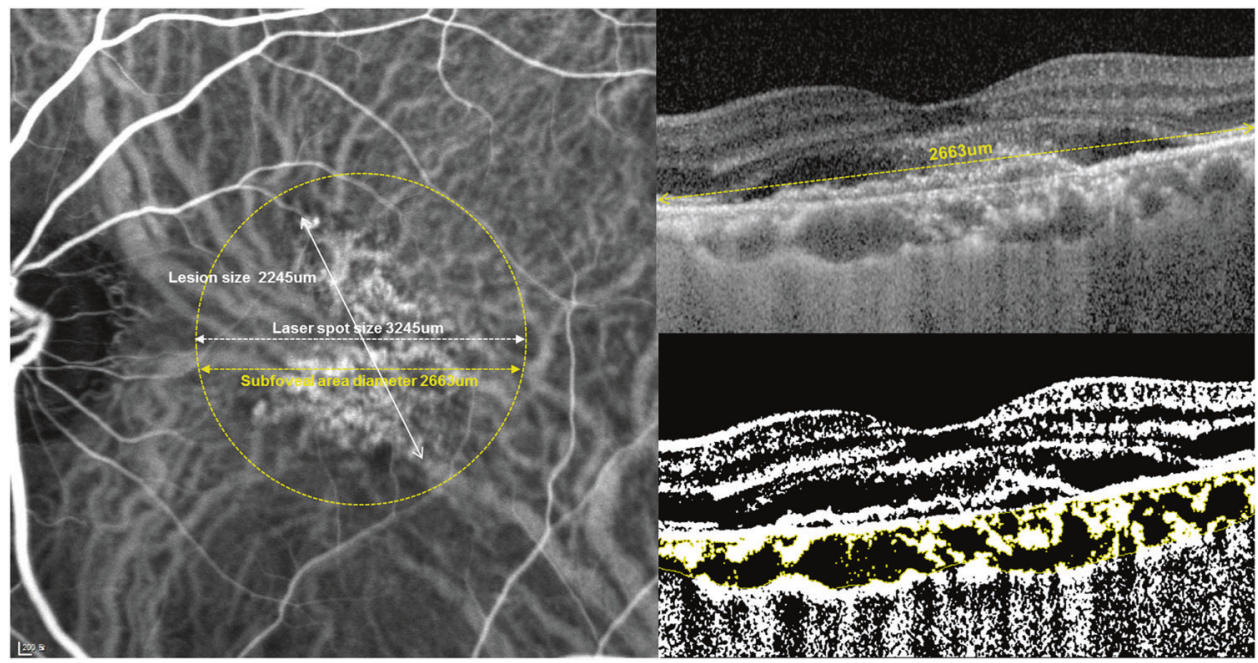

Fig. 1 Measurement of choroidal vascular density (Left) Indocyanine green angiography image is marked with photodynamic therapy (PDT) laser spot (yellow dot circle). (Right) Enhanced depth imaging OCT image (Top) and converted binary image (Bottom) of the subfoveal area in the circle of PDT laser spot. An EDI-OCT image of was converted to a binary image using the ImageJ software. The dark areas

Table 2. Total choroid, Haller's layer, and pachyvessel were significantly thicker in responders compared with non-responders and reductions in those thicknesses were greater in responders at 3 months after PDT. Choroidal vascularity was also higher in responders compared with non-responders and was decreased at 3 months after PDT in responders but not in non-responders. Choroidal thicknesses at baseline and their changes after PDT did not differ between recurrent and non-recurrent group

\section{Choroidal thickness changes after combination PDT by groups}

In responders, thickness of total choroid, choriocapillaris/ Sattler's layer, and Haller's layer at 3 months after PDT (263 \pm 108 (95\% confidence interval (CI): 238-289) $\mu \mathrm{m}$, $78 \pm(95 \%$ CI: $67-89) \mu \mathrm{m}$, and $186 \pm(95 \%$ CI: $168-203)$ $\mu \mathrm{m}$, respectively) was significantly decreased compared with baseline. In non-responders, total choroid thinning at 3 months after PDT was significant $(215 \pm 65$ (95\% CI: $181-250) \mu \mathrm{m}, \quad P=0.024)$, whereas reduction in thickness of each layer did not reach statistical significance (Fig. 2, left).

Reduction in total choroidal thickness was also observed in both recurrent and non-recurrent group (215 \pm 65 (95\% CI: $181-250) \mu \mathrm{m}$ and $284 \pm 130$ (95\% CI: 232-337) $\mu \mathrm{m}$, respectively). However, in layer-by-layer analysis, Haller's layer thinning in non-recurrent group was the only statistically significant change (Fig. 2, right). (luminal) of choroid were traced by the Niblack method. After adding the data of the distance of each pixel, the choroidal area, luminal area, and interstitial area were automatically calculated. The dark pixels were defined as the luminal area. The stromal area was calculated by subtracting luminal area from the total choroidal area

\section{Pachyvessel and choroidal vascularity change after combination PDT by groups}

Luminal diameter of pachyvessel was thicker and choroidal vascularity was higher at baseline and their reduction at 3 months was more prominent in responders compared with non-responders. The reduction of pachyvessel diameter was significant at 3 months after PDT in both responders and non-responders, whereas $\mathrm{CV}$ reduction was significant in responders but not in non-responders (Fig. 3, left).

Pachyvessel diameter and choroidal vascularity at baseline and their reduction at 3 months did not differ between non-recurrent and recurrent eyes. The reduction of pachyvessel diameter and choroidal vascularity at 3 months was significant in both recurrent and non-recurrent group. The thinning of pachyvessel and decreased vascularity was maintained after PDT in non-recurrent group, whereas a rebound increase in the pachyvessel diameter and vascularity was observed at the time of recurrence (Fig. 3, right).

\section{Discussion}

In this study, we analyzed the association of clinical factors, especially choroidal morphology, with anatomic response and recurrence after combination PDT in PCV. We observed that total choroid and Haller's layer thickness, pachyvessel diameter, hyperpermeability, choroidal vascularity, and changes in the choroidal thickness after treatment differed between responders and non-responders. The 
Table1 Comparison of baseline clinical characteristic between groups

\begin{tabular}{|c|c|c|c|c|c|c|}
\hline Characteristics & $\begin{array}{l}\text { Responders } \\
(n=70)\end{array}$ & $\begin{array}{l}\text { Non-responders } \\
(n=16)\end{array}$ & $P$ value & $\begin{array}{l}\text { Non-recurrent eyes } \\
(n=44)\end{array}$ & $\begin{array}{l}\text { Recurrent eyes } \\
(n=26)\end{array}$ & $P$ value \\
\hline \multicolumn{7}{|l|}{ Mean \pm SD (range) } \\
\hline Age (years) & $66.7 \pm 6.5(52-83)$ & $65.4 \pm 3.7(54-70)$ & $0.280^{\mathrm{a}}$ & $66.0 \pm 6.9(52-80)$ & $67.9 \pm 6.0(60-83)$ & $0.263^{\mathrm{a}}$ \\
\hline Baseline BCVA (LogMAR) & $0.39 \pm 0.33(0.00-1.70)$ & $0.51 \pm 0.29(0.10-1.10)$ & $0.191^{\mathrm{a}}$ & $0.43 \pm 0.37(0.00-1.70)$ & $0.34 \pm 0.26(0.00-0.90)$ & $0.291^{\mathrm{a}}$ \\
\hline Refractive error (diopter) & $0.13 \pm 1.36(-3.50-3.00)$ & $0.31 \pm 0.73(-1.00-1.25)$ & $0.601^{\mathrm{a}}$ & $-0.02 \pm 1.43(-3.50-3.00)$ & $0.38 \pm 1.24(-2.75-3.00)$ & $0.243^{\mathrm{a}}$ \\
\hline $\begin{array}{l}\text { Previous anti-VEGF } \\
\text { treatments }(n)\end{array}$ & $2.9 \pm 3.2(0-11)$ & $2.6 \pm 3.4(0-10)$ & $0.796^{\mathrm{a}}$ & $2.7 \pm 3.1(0-11)$ & $3.2 \pm 3.4(0-11)$ & $0.554^{\mathrm{a}}$ \\
\hline $\begin{array}{l}\text { Central macular thickness } \\
(\mu \mathrm{m}),\end{array}$ & $369 \pm 109(141-843)$ & $378 \pm 70(232-455)$ & $0.781^{\mathrm{a}}$ & $379 \pm 129(141-843)$ & $354 \pm 66(254-540)$ & $0.362^{\mathrm{a}}$ \\
\hline GLD of lesion $(\mu \mathrm{m})$ & $2458 \pm 737(1034-4735)$ & $2543 \pm 791(1276-4182)$ & $0.718^{\mathrm{a}}$ & $2403 \pm 821(1034-4735)$ & $2544 \pm 591(1515-4233)$ & $0.450^{\mathrm{b}}$ \\
\hline Number of polyps $(n)$ & $2.0 \pm 1.6(1-9)$ & $1.7 \pm 1.0(1-4)$ & $0.555^{\mathrm{a}}$ & $2.0 \pm 1.6(1-9)$ & $2.0 \pm 1.7(1-9)$ & $0.927^{\mathrm{a}}$ \\
\hline $\begin{array}{l}\text { Height of PED }(\mu \mathrm{m}) \\
n(\%)\end{array}$ & $247 \pm 172(0-805)$ & $224 \pm 111(0-471)$ & $0.623^{\mathrm{a}}$ & $272 \pm 173(0-805)$ & $209 \pm 167(0-601)$ & $0.337^{\mathrm{a}}$ \\
\hline Gender (male/female) & $49 / 21$ & $12 / 4$ & $0.750^{\mathrm{c}}$ & $28 / 16$ & $21 / 4$ & $0.073^{c}$ \\
\hline Treatment-naive eyes & $35(50)$ & $11(68)$ & $0.175^{\mathrm{c}}$ & $21(48)$ & $14(54)$ & $0.976^{\mathrm{c}}$ \\
\hline Choroidal hyperpermeability & $31(44)$ & $2(13)$ & $0.018^{\mathrm{c}}$ & $19(43)$ & $12(46)$ & $0.809^{\mathrm{c}}$ \\
\hline \multicolumn{7}{|l|}{ Presence of fluid } \\
\hline Subretinal & $69(99)$ & $15(94)$ & $0.248^{\mathrm{c}}$ & $44(100)$ & $25(96)$ & $0.190^{\mathrm{c}}$ \\
\hline Intraretinal & $12(17)$ & $0(0)$ & $0.086^{\mathrm{c}}$ & $8(18)$ & $4(27)$ & $0.668^{\mathrm{c}}$ \\
\hline PED & $60(86)$ & $13(81)$ & $0.706^{\mathrm{c}}$ & $38(86)$ & $22(85)$ & $0.293^{c}$ \\
\hline Lesion location & & & $0.782^{\mathrm{c}}$ & & & $0.498^{\mathrm{c}}$ \\
\hline Macula & $68(97)$ & $16(100)$ & & $43(98)$ & $25(96)$ & \\
\hline Peripapillary & $2(10)$ & $0(0)$ & & $1(2)$ & $1(4)$ & \\
\hline
\end{tabular}

$S D$ standard deviation, $B C V A$ best-corrected visual acuity, $V E G F$ vascular endothelial growth factor, $G L D$ greatest linear dimension identified by indocyanine angiography, $P E D$ pigment epithelial detachment.

${ }^{a}$ Mann-Whitney test between groups

${ }^{\mathrm{b}}$ Student's $t$-test between groups

${ }^{\mathrm{c}} \mathrm{Chi}$-square test between groups

change in Haller's layer thickness, pachyvessel diameter, and choroidal vascularity after treatment differed between recurrent and non-recurrent eyes.

The main therapeutic mechanism of PDT in AMD is ablation of CNV through selective uptake of photosensitizer by neovascular endothelial cells [26]. In addition, PDT causes collateral choriocapillaris damage and vascular remodeling in the underlying choroid [27-29]. The concept of choroidal vascular remodeling enabled application of PDT to patients with CSC, with the aim of decreasing vascular hyperpermeability in the area exposed to photoactivation [30]. Maruko et al. [31, 32] reported that subfoveal choroidal thickness decreases after PDT in CSC and PCV. They speculated that the choroidal thinning after PDT was a result of decreased choroidal hyperpermeability due to the vascular remodeling effect of PDT. In this study, we demonstrated that total choroidal thickness was significantly greater, hyperpermeability was more frequent, and reduction in choroidal thickness was more prominent in responders. This is in line with previous reports, supporting the notion that decreased hyperpermeability, and so decreased choroidal thickness, after PDT is related to treatment outcome.

In addition, our results revealed that responders had thicker Haller's layer and pachyvessel, which was also the area that showed significant difference in changes after PDT between the responders vs. non-responders and the recurrent vs. non-recurrent group. This suggests that the Haller's layer is related to the pathologic change and response to PDT in eyes with PCV. Exudation from choriocapillaris may accumulate in the interstitial stroma of the Haller's layer, resulting in thickening of the Haller's layer and attenuation of the overlying choriocapillaris layer. A greater decrease in the thickness of this layer may imply a greater effect of PDT on hyperpermeability that might have led to a better treatment outcome.

On the other hand, the Haller's layer is the area in which pachyvessel lies. Pachyvessel is increasingly noticed as a characteristic finding of pachychoroid spectrum diseases $[7,8$, 10]. The thickening of the choroid in eyes with CSC and PCV is known to be primarily associated with the thickening of the Haller vessels [33, 34]. Thinning of the Haller's layer after PDT can also result from constriction of pachyvessels, which was demonstrated by the changes of pachyvessel diameter in this study. The reduction of the pachyvessel diameter was significant after PDT and it increased again at the time of recurrence. This suggests that pachyvessel diameter might reflect disease activity. Furthermore, the baseline choroidal vascularity was higher in responders and reduction after PDT 
Table 2 Comparisons of baseline choroidal morphology and changes at 3 months after PDT between groups

\begin{tabular}{|c|c|c|c|c|}
\hline Time & Layer of choroid & $\begin{array}{l}\text { Mean } \pm \text { SD (range) } \\
\text { Responders }(n=70)\end{array}$ & $\begin{array}{l}\text { Mean } \pm \text { SD }(\text { range }) \\
\text { Non-responders }(n=16)\end{array}$ & $P$ value \\
\hline \multirow[t]{6}{*}{ Baseline } & Total choroidal thickness $(\mu \mathrm{m})$ & $297.6 \pm 111.2(100-554)$ & $226.9 \pm 56.0(109-330)$ & $0.001^{\mathrm{b}}$ \\
\hline & Choriocapillaris/Sattler's layer thickness $(\mu \mathrm{m})$ & $84.9 \pm 37.2(14-177)$ & $83.1 \pm 24.3(53-201)$ & $0.868^{\mathrm{c}}$ \\
\hline & Haller's layer thickness $(\mu \mathrm{m})$ & $212.7 \pm 87.5(75-388)$ & $143.8 \pm 50.7(56-276)$ & $<0.001^{\mathrm{b}}$ \\
\hline & Ratio $^{\text {a }}$ & $0.707 \pm 0.088(0.446-0.958)$ & $0.637 \pm 0.148(0.272-0.836)$ & $0.083^{\mathrm{b}}$ \\
\hline & Luminal diameter of pachyvessel $(\mu \mathrm{m})$ & $275.9 \pm 93.6(104-493)(n=66)$ & $209.6 \pm 31.0(169-275)(n=16)$ & $<0.001^{\mathrm{b}}$ \\
\hline & Choroidal vascularity & $0.682 \pm 0.064(0.524-0.812)$ & $0.596 \pm 0.049(0.514-0.690)$ & $<0.001^{\mathrm{c}}$ \\
\hline \multirow[t]{7}{*}{ Change } & Total choroidal thickness $(\mu \mathrm{m})$ & $-33.0 \pm 38.6(-126-+113)$ & $-10.4 \pm 19.1(-45-+32)$ & $0.036^{\mathrm{c}}$ \\
\hline & Choriocapillaris/Sattler's layer thickness $(\mu \mathrm{m})$ & $-7.19 \pm 24.3(-63-+71)$ & $-5.13 \pm 15.2(-32-+21)$ & $0.746^{\mathrm{c}}$ \\
\hline & Haller's layer thickness $(\mu \mathrm{m})$ & $-27.0 \pm 40.5(-118-+74)$ & $-6.4 \pm 24.3(-60-+37)$ & $0.012^{\mathrm{c}}$ \\
\hline & Ratio $^{\mathrm{a}}$ & $0.006 \pm 0.95(-0.198-+0.271)$ & $0.004 \pm 0.082(-0.131-+0.150)$ & $0.946^{\mathrm{c}}$ \\
\hline & Luminal diameter of pachyvessel $(\mu \mathrm{m})$ & $-60.3 \pm 40.6(-195-+38)(n=66)$ & $-20.1 \pm 25.2(-84-+18)(n=16)$ & $<0.001^{\mathrm{c}}$ \\
\hline & Choroidal vascularity & $-0.058 \pm 0.049(-0.280-0.026)$ & $0.004 \pm 0.039(-0.080-0.082)$ & $<0.001^{\mathrm{c}}$ \\
\hline & & Non-recurrent eyes $(n=44)$ & Recurrent eyes $(n=26)$ & \\
\hline \multirow[t]{6}{*}{ Baseline } & Total choroidal thickness $(\mu \mathrm{m})$ & $290.1 \pm 99.3(100-479)$ & $310.3 \pm 130.1(155-554)$ & $0.500^{\mathrm{b}}$ \\
\hline & Choriocapillaris/Sattler's layer thickness $(\mu \mathrm{m})$ & $80.8 \pm 36.9(14-177)$ & $91.7 \pm 37.5(31-167)$ & $0.240^{\mathrm{c}}$ \\
\hline & Haller's layer thickness $(\mu \mathrm{m})$ & $209.3 \pm 78.8(76-375)$ & $218.5 \pm 101.9(75-388)$ & $0.693^{\mathrm{b}}$ \\
\hline & Ratio $^{\mathrm{a}}$ & $0.718 \pm 0.082(0.556-0.958)$ & $0.689 \pm 0.096(0.446-0.830)$ & $0.176^{\mathrm{c}}$ \\
\hline & Luminal diameter of pachyvessel $(\mu \mathrm{m})$ & $267.6 \pm 86.4(104-493)(n=41)$ & $289.5 \pm 104.8(121-473)(n=25)$ & $0.385^{\mathrm{c}}$ \\
\hline & Choroidal vascularity & $0.685 \pm 0.066(0.524-0.812)$ & $0.676 \pm 0.064(0.557-0.782)$ & $0.557^{\mathrm{c}}$ \\
\hline \multirow[t]{6}{*}{ Change } & Total choroidal thickness $(\mu \mathrm{m})$ & $-37.5 \pm 28.5(-126-+2)$ & $-25.9 \pm 50.4(-125-+113)$ & $0.294^{\mathrm{c}}$ \\
\hline & Choriocapillaris/Sattler's layer thickness $(\mu \mathrm{m})$ & $-7.2 \pm 24.5(-63-+71)$ & $-7.2 \pm 24.5(-51-+44)$ & $0.993^{\mathrm{b}}$ \\
\hline & Haller's layer thickness $(\mu \mathrm{m})$ & $-31.8 \pm 34.3(-118-+37)$ & $-18.8 \pm 48.9(-117-+74)$ & $0.195^{\mathrm{c}}$ \\
\hline & Ratio $^{\mathrm{a}}$ & $-0.002 \pm 0.092(-0.198-+0.261)$ & $0.020 \pm 0.099(-0.103-+0.271)$ & $0.341^{\mathrm{b}}$ \\
\hline & Luminal diameter of pachyvessel $(\mu \mathrm{m})$ & $-61.2 \pm 39.3(-195--2)(n=41)$ & $-58.7 \pm 43.4(-128-+38)(n=25)$ & $0.808^{\mathrm{c}}$ \\
\hline & Choroidal vascularity & $-0.049 \pm 0.042(-0.142-0.026)$ & $-0.073 \pm 0.058(-0.281--0.003)$ & $0.097^{\mathrm{c}}$ \\
\hline
\end{tabular}

$S D$ standard deviation

a'Ratio of Haller's layer thickness to total choroidal thickness

b'Student's $t$-test between groups

${ }^{\mathrm{c}}$ Mann-Whitney test between groups

was more prominent in responders. Also, a rebound increase of choroidal vascularity was observed at the time of recurrence. Although the total choroidal thickness can change owing to changes in stroma, our results suggest that the changes in the vascular area are more prominent.

We assume that the reason for insufficient response to PDT in non-responders is that the main pathogenic mechanism in those eyes may be somewhat different from responders. In non-responders, pathogenetic processes involving ischemia and VEGF production may be the major mechanism for disease development and progression as in typical AMD. Kim et al. [35] reported that a thin choroid served as a good prognostic factor for anti-VEGF treatment in eyes with PCV. And in our previous study, aqueous VEGF level was higher in PCV eyes with thinner choroid [36]. These previous reports support the hypothesis that VEGF upregulation plays a large role in eyes with PCV with a thinner choroid, which were poor responders to PDT.
No significant thinning of the choriocapillaris/Sattler's or Haller's layer after PDT in layer-by-layer analysis was found in eyes with recurrence. This may mean that PDT had lesser effects on CNV itself, choroidal hyperpermeability, or pachyvessel in those eyes. We cannot rule out the possibility of undertreatment which might have caused a recurrence within a short period.

There are several limitations to our study owing to its retrospective study design. First, the first-line treatment was not controlled and both treatment-naive eyes and eyes with history of anti-VEGF treatment were included in the study cohort. However, history and numbers of anti-VEGF treatment did not affect the outcome of combination PDT in both responsiveness and recurrence. Nonetheless, there is still a possibility for selection bias, as some cases that did well on anti-VEGF monotherapy might have been excluded from the study. Second, responsiveness and recurrence were defined only anatomically with resolution of fluid on OCT. It should 


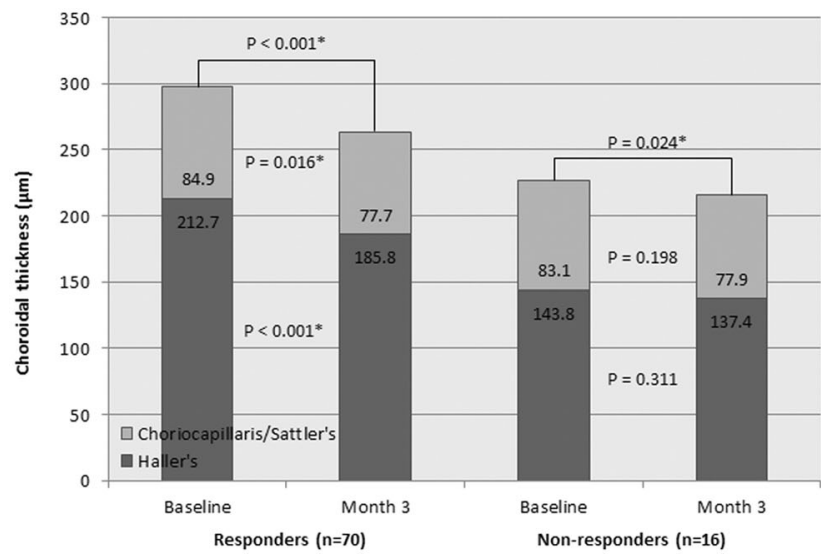

Fig. 2 Changes of subfoveal choroidal thickness at month 3 after combination PDT compared with baseline: responders vs. nonresponders and non-recurrent vs. recurrent eyes. (Left) Thinning of total choroid was observed in both responders and non-responders at 3 months after combination photodynamic therapy (PDT). Reduction in thickness of choriocapillaris/Sattler's layer and Haller's layer at 3 months after PDT was significant in responders, but no such change

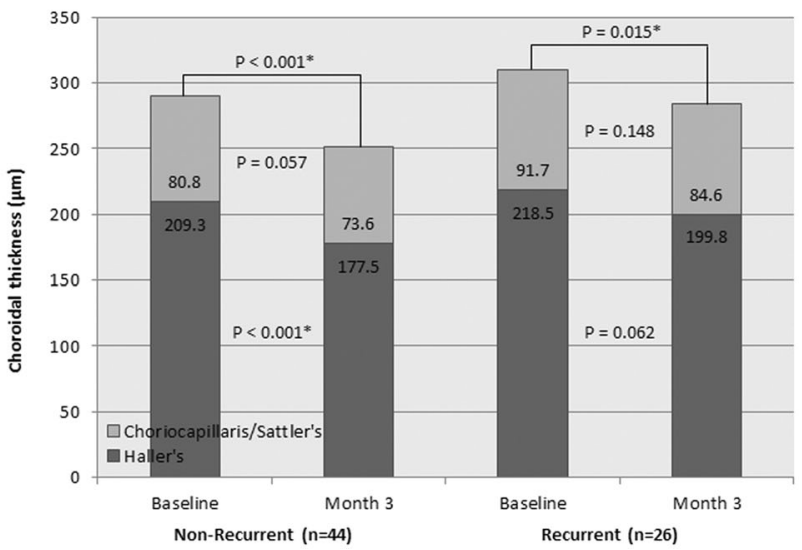

was observed in non-responders. (Right) Thinning of total choroidal was observed also in both recurrent and non-recurrent eyes at 3 months after combination PDT. Reduction in Haller's layer thickness in nonrecurrent eyes was statistically significant whereas no significant reduction was observed in recurrent eyes in layer-by-layer analysis. *Statistically significant $P$ value using paired $t$ test

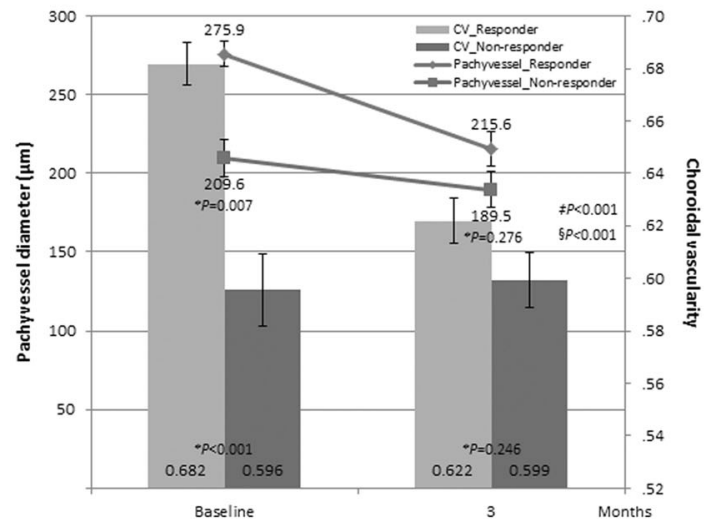

Fig. 3 Comparison of pachyvessel diameter and choroidal vascularity change between responders vs. non-responders and non-recurrent vs. recurrent eyes during 1-year follow-up Polygonal lines demonstrate pachyvessel diameter change and bar graphs indicate choroidal vascularity (CV). (Left) Reduction of pachyvessel diameter was significant at 3 months after photodynamic therapy (PDT) in both responders and non-responders $(P<0.001$ and $=0.006$, respectively, paired $t$ test). $\mathrm{CV}$ reduction at 3 months was significant in responders but not in non-responders $(P<0.001$ and $=0.728)$. (Right) In recurrent eyes, pachyvessel diameter increased at recurrence $(P=0.047$ compared with a previous measurement), whereas non-recurrent eyes maintained thinning of the pachyvessel until 12 months $(P<0.001,=$

be considered that a favorable anatomical outcome does not ensure a good visual outcome. Nevertheless, using anatomical changes to assess the treatment response seems appropriate because the resolution of exudation is directly related to the mechanism of action of treatment. Third, there could have been a selection bias caused by excluding eyes that developed massive hemorrhage after PDT and that received the second PDT within 1 year, although the numbers were small. In addition, initial anti-VEGF treatment before PDT could have

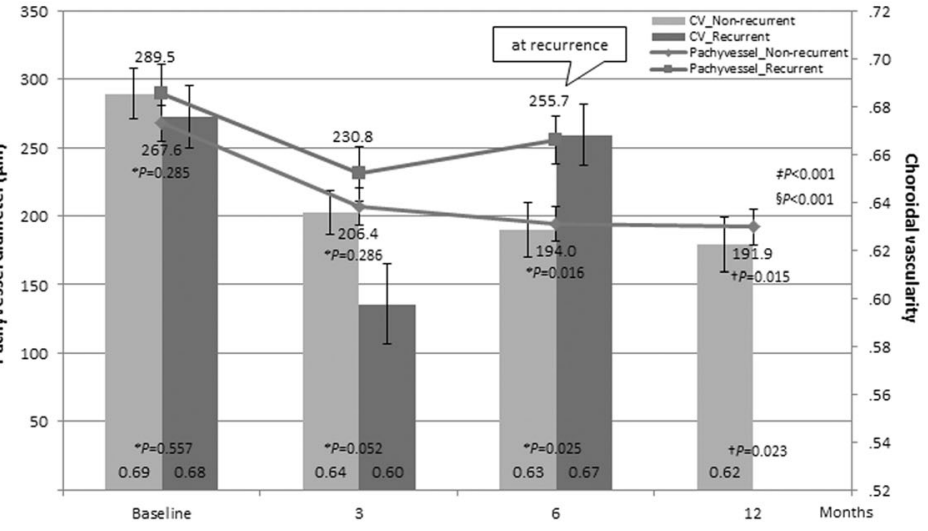

0.218 , and 0.816 compared with previous measurements, respectively). Pachyvessel at recurrence in recurrent group was thicker compared with that of 6 months and 12 months after PDT in nonrecurrent eyes. $\mathrm{CV}$ also increased at the time of recurrence in recurrent eyes $(P=0.001$ compared with a previous measurement), whereas it decreased continuously in non-recurrent eyes $(P<0.001,=0.011$, and 0.017 compared with previous measurements, respectively). CV: Choroidal vascularity. *Student $t$ test between groups ${ }^{\dagger}$ Student $t$ test compared with recurrence group at the time of recurrence. ${ }^{*}$ Repeatedmeasures analysis of variance interaction for pachyvessel diameter ${ }^{\S}$ Repeated-measures analysis of variance interaction for CV. Error bar indicates standard error

affected the decrease in choroidal thickness. However, we believe the degree would be negligible. One-time injection of bevacizumab is known to cause no significant change in choroidal thickness [37]. Despite the aforementioned limitations, this is the first study that analyzed choroid by layers and under the site of pathology in vivo in PCV eyes undergoing combination PDT.

In summary, thicker baseline subfoveal choroid, Haller's layer, and pachyvessel along with higher choroidal 
vascularity and hyperpermeability, and prominent reduction after treatment were predictive factors for good short-term response. A rebound increase in pachyvessel diameter and choroidal vascularity was observed in eyes with recurrence. These findings indicate that choroidal features reflect the underlying pathophysiology of PCV regarding the action mechanism of PDT, thereby leading to different treatment outcomes. Results of this study will facilitate prediction of the outcomes of combination PDT treatment and determination of the optimal treatment strategy for patients with PCV.

\section{Summary}

\section{What was known before}

- Clinical characteristics and treatment outcome of eyes with PCV is not uniform.

- Choroidal morphology seems to be strongly associated with pathogenesis of PCV and treatment response.

\section{What this study adds}

- We found that characteristic features of pachychoroid (i.e., increased choroidal thickness, Haller's layer proportion, and pachyvessel diameter) and high vascularity are positive predictive factors for combination PDT in eyes with PCV.

- The results of this study will facilitate determination of the optimal treatment for patients with PCV in which both anti-VEGF and PDT are viable treatment options.

Funding There is no funding or conflicts of interest relating to any author relating to this article. Won Ki Lee has served on advisory boards for Novartis Bayer, Allergan, Alcon, and Santen, and has received consultancy fees from these companies. He has received payments for lectures from Novartis, Bayer, Allergan, and Alcon. Jiwon Baek, Jae Hyung Lee, and Sohee Jeon have no financial disclosure to report. The authors indicate no funding or financial support.

\section{Compliance with ethical standards}

Conflict of interest The authors declare that they have no conflict of interest.

\section{References}

1. Yannuzzi LA, Sorenson J, Spaide RF, Lipson B. Idiopathic polypoidal choroidal vasculopathy (IPCV). Retina. 1990;10:1-8.

2. Spaide RF, Yannuzzi LA, Slakter JS, Sorenson J, Orlach DA. Indocyanine green videoangiography of idiopathic polypoidal choroidal vasculopathy. Retina. 1995;15:100-10.
3. Ojima Y, Hangai M, Sakamoto A, Tsujikawa A, Otani A, Tamura $\mathrm{H}$, et al. Improved visualization of polypoidal choroidal vasculopathy lesions using spectral-domain optical coherence tomography. Retina. 2009;29:52-9.

4. Chung SE, Kang SW, Lee JH, Kim YT. Choroidal thickness in polypoidal choroidal vasculopathy and exudative age-related macular degeneration. Ophthalmology. 2011;118:840-5.

5. Koizumi H, Yamagishi T, Yamazaki T, Kawasaki R, Kinoshita S. Subfoveal choroidal thickness in typical age-related macular degeneration and polypoidal choroidal vasculopathy. Graefes Arch Clin Exp Ophthalmol. 2011;249:1123-8.

6. Jirarattanasopa P, Ooto S, Nakata I, Tsujikawa A, Yamashiro K, Oishi A, et al. Choroidal thickness, vascular hyperpermeability, and complement factor $\mathrm{H}$ in age-related macular degeneration and polypoidal choroidal vasculopathy. Invest Ophthalmol Vis Sci. 2012;53:3663-72.

7. Alasil T, Ferrara D, Adhi M, Brewer E, Kraus MF, Baumal CR, et al. En face imaging of the choroid in polypoidal choroidal vasculopathy using swept-source optical coherence tomography. Am J Ophthalmol. 2015;159:634-43.

8. Dansingani KK, Balaratnasingam C, Naysan J, Freund KB. En face imaging of pachychoroid spectrum disorders with sweptsource optical coherence tomography. Retina. 2016;36:499-516.

9. Pang CE, Freund KB. Pachychoroid neovasculopathy. Retina. 2015;35:1-9.

10. Lee WK, Baek J, Dansingani KK, Lee JH, Freund KB. Choroidal morphology in eyes with polypoidal choroidal vasculopathy and normal or subnormal subfoveal choroidal thickness. Retina. 2016;36:S73-82.

11. Kawamura A, Yuzawa M, Mori R, Haruyama M, Tanaka K. Indocyanine green angiographic and optical coherence tomographic findings support classification of polypoidal choroidal vasculopathy into two types. Acta Ophthalmol. 2013;91:e474-81.

12. Coscas G, Lupidi M, Coscas F, Benjelloun F, Zerbib J, Dirani A, et al. Toward a specific classification of polypoidal choroidal vasculopathy: idiopathic disease or subtype of age-related macular degeneration. Invest Ophthalmol Vis Sci. 2015;56:3187-95.

13. Koizumi H, Yamagishi T, Yamazaki T, Kinoshita S. Relationship between clinical characteristics of polypoidal choroidal vasculopathy and choroidal vascular hyperpermeability. Am J Ophthalmol. 2013;155:305-.e301.

14. Sonoda S, Sakamoto T, Otsuka H, Yoshinaga N, Yamashita T, Ki IY, et al. Responsiveness of eyes with polypoidal choroidal vasculopathy with choroidal hyperpermeability to intravitreal ranibizumab. BMC Ophthalmol. 2013;13:43.

15. Cho HJ, Kim HS, Jang YS, Han JI, Lew YJ, Lee TG, et al. Effects of choroidal vascular hyperpermeability on anti-vascular endothelial growth factor treatment for polypoidal choroidal vasculopathy. Am J Ophthalmol. 2013;156:1192-200 e1191.

16. Stangos AN, Gandhi JS, Nair-Sahni J, Heimann H, Pournaras CJ, Harding SP. Polypoidal choroidal vasculopathy masquerading as neovascular age-related macular degeneration refractory to ranibizumab. Am J Ophthalmol. 2010;150:666-73.

17. Cho M, Barbazetto IA, Freund KB. Refractory neovascular agerelated macular degeneration secondary to polypoidal choroidal vasculopathy. Am J Ophthalmol. 2009;148:70-8.e71.

18. Hatz K, Prunte C. Polypoidal choroidal vasculopathy in Caucasian patients with presumed neovascular age-related macular degeneration and poor ranibizumab response. $\mathrm{Br} \mathrm{J}$ Ophthalmol. 2014;98:188-94.

19. Ozkaya A, Alagoz C, Garip R, Alkin Z, Perente I, Yazici AT et al. The role of indocyanine green angiography imaging in further differential diagnosis of patients with nAMD who are morphologically poor responders to ranibizumab in a real-life setting. Eye (Lond). 2016;30:958-65. 
20. Koh A, Lee WK, Chen LJ, Chen SJ, Hashad Y, Kim H, et al. EVEREST study: efficacy and safety of verteporfin photodynamic therapy in combination with ranibizumab or alone versus ranibizumab monotherapy in patients with symptomatic macular polypoidal choroidal vasculopathy. Retina. 2012;32:1453-64.

21. Tatar O, Shinoda K, Adam A, Eckert T, Eckardt C, Lucke K, et al. Effect of verteporfin photodynamic therapy on endostatin and angiogenesis in human choroidal neovascular membranes. $\mathrm{Br} \mathbf{J}$ Ophthalmol. 2007;91:166-73.

22. Staurenghi G, Sadda S, Chakravarthy U, Spaide RF. Proposed lexicon for anatomic landmarks in normal posterior segment spectral-domain optical coherence tomography: the IN*OCT consensus. Ophthalmology. 2014;121:1572-8.

23. Guyer DR, Yannuzzi LA, Slakter JS, Sorenson JA, Hope-Ross M, Orlock DR. Digital indocyanine-green videoangiography of occult choroidal neovascularization. Ophthalmology. 1994;101:1727-35.

24. Branchini LA, Adhi M, Regatieri CV, Nandakumar N, Liu JJ, Laver N, et al. Analysis of choroidal morphologic features and vasculature in healthy eyes using spectral-domain optical coherence tomography. Ophthalmology. 2013;120:1901-8.

25. Sonoda S, Sakamoto T, Yamashita T, Shirasawa M, Uchino E, Terasaki H, et al. Choroidal structure in normal eyes and after photodynamic therapy determined by binarization of optical coherence tomographic images. Invest Ophthalmol Vis Sci. 2014;55:3893-9.

26. Schmidt-Erfurth U, Hasan T. Mechanisms of action of photodynamic therapy with verteporfin for the treatment of age-related macular degeneration. Surv Ophthalmol. 2000;45:195-214.

27. Schmidt-Erfurth U, Michels S, Barbazetto I, Laqua H. Photodynamic effects on choroidal neovascularization and physiological choroid. Invest Ophthalmol Vis Sci. 2002;43: $830-41$

28. Schlotzer-Schrehardt U, Viestenz A, Naumann GO, Laqua H, Michels S, Schmidt-Erfurth U. Dose-related structural effects of photodynamic therapy on choroidal and retinal structures of human eyes. Graefes Arch Clin Exp Ophthalmol. 2002;240: $748-57$.
29. Isola V, Pece A, Brancato R. Circulatory changes in the choroidal vasculature after verteporfin-based photodynamic therapy for choroidal neovascularization in age-related macular degeneration. Retina. 2004;24:618-20.

30. Yannuzzi LA, Slakter JS, Gross NE, Spaide RF, Costa D, Huang $\mathrm{SJ}$, et al. Indocyanine green angiography-guided photodynamic therapy for treatment of chronic central serous chorioretinopathy: a pilot study. Retina. 2003;23:288-98.

31. Maruko I, Iida T, Oyamada H, Sugano Y, Ojima A, Sekiryu T. Choroidal thickness changes after intravitreal ranibizumab and photodynamic therapy in recurrent polypoidal choroidal vasculopathy. Am J Ophthalmol. 2013;156:548-56.

32. Teo KYC, Yanagi Y, Lee SY, Yeo IYS, Tan GSW, Mathur R et al. Comparison of optical coherence tomography angiographic changes after anti-vascular endothelial growth factor therapy alone or in combination with photodynamic therapy in polypoidal choroidal vasculopathy. Retina. 2018;38:1675-87.

33. Kuroda Y, Ooto S, Yamashiro K, Oishi A, Nakanishi H, Tamura $\mathrm{H}$ et al. Increased choroidal vascularity in central serous chorioretinopathy quantified using swept-source optical coherence tomography. Am J Ophthalmol. 2016;169:199-07.

34. Wei C, Liu R, Li J, Yang Y, Ding X. Choroidal analysis of polypoidal choroidal vasculopathy by spectral domain optical coherence tomography. Eye Sci. 2014;29:20-24.

35. Kim H, Lee SC, Kwon KY, Lee JH, Koh HJ, Byeon SH et al. Subfoveal choroidal thickness as a predictor of treatment response to anti-vascular endothelial growth factor therapy for polypoidal choroidal vasculopathy. Graefes Arch Clin Exp Ophthalmol. 2016; 254:1497-03.

36. Baek J, Lee JH, Lee WK. Clinical relevance of aqueous vascular endothelial growth factor levels in polypoidal choroidal vasculopathy. Retina. 2017;37:943-50.

37. Sizmaz S, Kucukerdonmez C, Kal A, Pinarci EY, Canan H, Yilmaz G. Retinal and choroidal thickness changes after single anti-VEGF injection in neovascular age-related macular degeneration: ranibizumab vs bevacizumab. Eur J Ophthalmol. 2014;24:904-10. 